\title{
Emergency Left Colon Resection for Acute Perforation. Primary Anastomosis or Hartmann's Procedure? A Case-matched Control Study
}

\author{
Stefan Breitenstein - Armin Kraus · Dieter Hahnloser • Marco Decurtins • \\ Pierre-Alain Clavien · Nicolas Demartines
}

Published online: 24 August 2007

(C) Société Internationale de Chirurgie 2007

\begin{abstract}
Background The optimal treatment remains controversial for acute left-sided colon perforation. Therefore, the effectiveness and safety of primary anastomosis versus Hartmann's operation (HP) was compared in a case-matched control study.

Methods Thirty consecutive patients with primary anastomosis and protective ileostomy (PAS) were matched to 30 HP patients, controlling for age, gender, American Society of Anesthesiologists (ASA) score, body mass index (BMI), and peritonitis severity (Hinchey). In a second analysis, PAS patients with purulent peritonitis (Hinchey 3 ) were matched to patients with primary anastomosis without ileostomy (PA).

Results Hospital mortality was similar between HP (17\%) and PAS (10\%). Complication frequency and severity (requiring re-intervention or admission to the Intensive Care Unit [ICU]) were comparable for the first operation $(60 \%$ versus $56 \%$ and $30 \%$ versus $32 \%)$. The stoma reversal rate was higher in PAS than in HP $(96 \%$ versus $60 \%, p=0.001)$, with significantly fewer complications (23\% versus $66 \%, p=0.02$ ), and lower severity ( $7 \%$ versus $33 \%, p=0.02$ ). Additional analysis of PAS versus PA
\end{abstract}

Presented at the 13th Annual Meeting of the European Surgical Association (ESA), Zurich, 2006.

S. Breitenstein $(\bowtie) \cdot$ A. Kraus · D. Hahnloser · P.-A. Clavien . N. Demartines

Department of Visceral and Transplantation Surgery, University

Hospital, Ramistrasse 100, CH-8091 Zurich, Switzerland

e-mail: stefan.breitenstein@usz.ch

M. Decurtins

Department of Surgery, Kantonsspital Winterthur, Brauerstrasse

15, Postfach 834, CH-8401 Winterthur, Switzerland showed similar morbidity $(52 \%$ versus $41 \%, p=0.45)$ and complication severity $(18 \%$ versus $24 \%, p=0.51)$, whereas overall operation time and hospital stay were significantly shorter in PA (169 versus $320 \mathrm{~min}, p=0.003$, 17 versus 28 days, $p<0.001)$.

Conclusions Primary anastomosis and protective ileostomy is a superior treatment to HP in acute left-sided colon perforation. In the absence of feculent peritonitis an ileostomy appears unnecessary.

How to manage acute left-sided colon perforation remains controversial, as no study providing a high level of evidence is currently available. Although the superiority of an approach with primary resection has been established in multicenter randomized studies over the traditional threestage treatment (diversion, resection, and reanastomosis) [1], the indication to perform a primary anastomosis versus an end-colostomy (Hartmann's procedure; HP) is still under debate.

Acute colonic perforation, mostly caused by diverticulitis [2-4], is associated with high rates of mortality (up to $20 \%$ ) and morbidity (up to 60\%) $[3,5]$. The prevalence of diverticular disease is increasing in Western countries, as approximately one-third of the population is affected by the sixth decade of life, and half by the ninth decade [6, 7]. Diverticulitis occurs in $10 \%-25 \%$ of patients with diverticulosis [8], and 10\%-20\% of those who are hospitalized require an urgent operation [9].

According to the Guidelines of the American Society of Colon and Rectal Surgeons, perforated diverticulitis with purulent or fecal peritonitis requires a HP [8]. The European Association of Endoscopic Surgeons recommends both primary anastomosis with protective stoma (PAS) and 
HP as treatment options for colon perforation with purulent peritonitis (Hinchey 3), whereas fecal peritonitis (Hinchey 4) should be treated only by HP. Both recommendations are based on consensus conferences. A comprehensive review demonstrated that PAS may compare favorably to HP with respect to mortality and complications [10]. However, the reports include a considerable patient selection bias, and conclusions must therefore be taken with caution. Recently, it has been shown that primary anastomosis is possible in cases of free perforation with generalized peritonitis [3, 11-14], but it remains unclear whether a diverting stoma is necessary, particularly in the presence of purulent peritonitis [7, 15].

In the absence of any randomized trial, the next best approach to addressing whether PAS is superior to HP in patients with acute left-sided colon perforation is a casematched control study. We therefore conducted such a study, with the primary aim to compare the effectiveness and safety of PAS versus HP in acute left-sided colon perforation. A secondary aim was to evaluate the necessity of a diverting ileostomy in the case of primary anastomosis.

\section{Materials and methods}

Between January 2000 and July 2005, 110 patients (45 HP, 30 PAS, 35 primary anastomosis without protective stoma) underwent operation for acute left-sided colon perforation at two institutions (an academic medical center and a large community hospital). A total of 30 patients underwent PAS, and they were matched one-to-one with $30 \mathrm{HP}$ patients, controlling for age $(<65$ years, 65-75 years, $>75$ years), gender, American society of Anesthesiologists (ASA) score (ASA grade \pm 1 ), body mass index (BMI; \pm 5 $\mathrm{kg} / \mathrm{m}^{2}$ ) and peritonitis severity classification by Hinchey stage (1 and 2 versus 3 and 4) [16].

During the same time period, 35 patients underwent a primary anastomosis but without protective stoma (PA) and were matched one-to-one to the PAS group according to the same matching criteria. All patients were matched before outcome mesures were reviewed.

Choice of surgical procedure and surgical technique

All patients had radiologically confirmed perforation of the left-sided colon and underwent emergency surgery. The choice of PAS versus HP versus PA was left to the clinical appreciation of the respective surgeons and was based on the prevailing strategy at the affiliated institution. All patients underwent open rectosigmoid resection. In the HP group an end-colostomy was performed on the left abdominal side. Stoma reversal was scheduled on the basis of a patient's general state of health and guidelines of the respective hospital. For stoma reversal, a midline incision and a colorectal transanal circular stapler anastomosis was carried out without colonic preparation. In the PAS and PA groups, rectosigmoid resection was followed by immediate colorectal anastomosis with the use of circular stapling. Intraoperative colon lavage was not routinely performed. The decision for protective loop ileostomy was at the discretion of the surgeon. Prior to ileostomy closure (segmental resection and hand-sewn anastomosis) anastomotic leakage or stenosis were routinely excluded by Gastrographin $($ e enema.

\section{Outcome measures}

Database evaluation and chart review were performed to extract data about intraoperative and postoperative parameters. Peritonitis was graded according to the Hinchey score, but the Mannheim Peritonitis score [17] and the APACHE II score [18] were also assessed. The number of immunosuppressed patients (due to organ transplantation versus other reasons) was recorded. Endpoints of the study were mortality, morbidity, and stoma reversal rates. Complications were graded according to a validated therapy-oriented complication score on a five-point scale [19]. Severe complications were defined as an event requiring intervention under local or general anesthesia or treatment in the intensive care unit (complication grades 3 and 4). When more than one complication occurred in a patient, only the most severe was taken into account for calculation of the complication grade in that patient. All data were collected individually for both the resection operation and the stoma reversal operation.

Statistical analysis

Statistical analysis was performed using standard software (SPSS 12.0 for Windows). Because of the matched arrangement of the data, data pairs were regarded as dependent. The Wilcoxon signed rank test was performed to compare continuous variables between two groups. Categorical variables were compared using the $\chi^{2}$ test according to McNemar. The sign test was performed for ordinal variables. Results of continuous parameters are expressed as mean \pm standard deviation, whereas discrete variables are expressed in mean and range. A $p$ value less than 0.05 was considered indicative of statistical significance. Hospitalization time was compared by the log rank test, with patients who died during the period of hospitalization rated as censored. 
Table 1 Demographics of patients undergoing Hartmann's procedure (HP) or primary anastomosis with protective ileostomy (PAS)

\begin{tabular}{llll}
\hline & $\begin{array}{l}\text { HP } \\
(n=30)\end{array}$ & $\begin{array}{l}\text { PAS } \\
(n=30)\end{array}$ & $p$ Value \\
\hline Age (years) & $65 \pm 14.4$ & $64 \pm 14.4$ & 0.67 \\
Gender (male/female) & $12 / 18$ & $10 / 20$ & 0.39 \\
BMI $\left(\mathrm{kg} / \mathrm{m}^{2}\right)$ & $25.7 \pm 4.1$ & $26.3 \pm 4.4$ & 0.33 \\
ASA I & 2 & 0 & \\
II & 6 & 8 & \\
III & 12 & 14 & \\
IV & 10 & 8 & \\
Hinchey stage 1 & 0 & 0 & \\
2 & 3 & 3 & \\
3 & 17 & 17 & \\
4 & 10 & 10 & 0.17 \\
MPI score & $23(6-34)$ & $21(9-32)$ & 0.65 \\
APACHE II & $12(2-28)$ & $13(1-23)$ & 0.61 \\
Immunosuppressed patients & $2 / 8$ & $0 / 8$ & \\
$\quad$ (transplantation/other) & & & \\
Etiology of perforation & $26 / 2 / 2$ & $26 / 3 / 1$ & 1.0 \\
$\quad$ diverticulitis/tumor/other) & & & \\
Number of patients & $24 / 6$ & $8 / 22$ & \\
$\quad$ per institution & & & \\
\hline
\end{tabular}

Data represent number of patients: mean \pm standard deviation for continuous data and median (range) of discrete data

$B M I$ body mass index; ASA American Society of Anesthesiologists; MPI Mannheim Peritonitis Index

\section{Results}

According to the study protocol, all patients were wellmatched for age, gender, ASA score, BMI, and peritonitis severity classified by Hinchey stage. Moreover the severity of the acute infection, measured by the Mannheim Peritonitis Index (MPI) [17] and APACHE II score [18], as well as the number of immunosuppressed patients (10 versus 8 ) and the etiology of perforation (26 patients with diverticulitis in each group) were comparable (Table 1).

Emergency resection operation: are there differences in mortality and morbidity between HP and PAS?

Hospital mortality was observed exclusively during the initial resection operation and was comparable between the groups (HP: 5 deaths, PAS: 3 deaths; $p=0.69$ ). Causes of death in the HP group were sepsis $(n=3)$, adult respiratory distress syndrome (ARDS: $n=1$ ), and cardiac failure $(n=1)$. In the PAS group, patients died from ARDS, pneumonia, or stroke $(n=1$ each). The total number of patients with complications (HP: 18 versus PAS: 17) and the severity of complications (including grade $5=$ death) were similar between the groups $(p=0.83$; Table 2$)$.
Surgical and nonsurgical complications in surviving patients were also similar in the two groups (10 cases each). In the HP group the following surgical complications were observed: wound infections $(n=4)$, intraabdominal infections $(n=4)$, stoma necrosis $(n=1)$, and bleeding $(n=1)$. In the PAS patients, the following complications were observed: wound infections $(n=3)$, intraabdominal infections $(n=4)$, leakages $(n=2)$, and stoma necrosis $(n=1)$. Six of 10 surgical complications in the HP group and in the PAS group, required surgical intervention (complication grade 3 or 4 ). The total number of nonsurgical complications was 10 in HP patients and 9 in PAS patients. In the HP group: thrombosis $(n=1)$, ARDS $(n=1)$, urinary tract infection $(n=1)$, reversible renal insufficiency $(n=2)$, cardiac complications $(n=3)$, gastric ulcer $(n=1)$, and central venous line infection $(n=1)$ were noted. In the PAS group: urinary tract infections $(n=3)$, reversible renal insufficiency $(n=1)$, cardiac failure $(n=1)$, reversible multi-organ failure $(n=1)$, gastric ulcer $(n=1)$, catheter infection $(n=1)$, and pleural effusion $(n=1)$ were recorded.

Operative time was significantly longer in the PAS group than in the HP group, and blood loss significantly higher, but these findings had no influence on the length of hospital stay (Table 2).

Stoma reversal operation: are there differences between HP and PAS concerning reversal rates, time to reversal, and complications?

Stoma reversal was performed significantly more frequently $(96 \%$ versus $60 \% ; p=0.001)$ and earlier (mean after 68 days versus 193 days; $p=0.001$ ) in the PAS group than in the HP group, and it was also associated with fewer complications (Table 2). Furthermore, complications were significantly less severe in PAS patients. Seven surgical complications occurred in the HP group and five in the PAS group. In the HP group: wound infections $(n=3)$, intra-abdominal infection $(n=1)$, leakage $(n=1)$, and a nonspecified complication $(n=1)$ were noted, whereas in the PAS group, they were wound infections $(n=2)$, leakage $(n=2)$, and ileus $(n=1)$. The total number of nonsurgical complications was six in the HP group, pleural effusion $(n=1)$, pulmonary embolism $(n=1)$, urinary tract infection $(n=1)$, myocardial infarction $(n=1)$, and others nonspecified $(n=2)$; and two in the PAS group, urinary tract infection $(n=1)$ and atrial fibrillation $(n=1)$. The operative time was significantly shorter, blood loss was less, and hospital stay was shorter in the PAS group (Table 2).

Analyzing the two procedures together (resection plus reversal operation) operative time $(395 \pm 103 \mathrm{~min}$ versus 
Table 2 Results of patients undergoing Hartmann's procedure (HP) or primary anastomosis with protective ileostomy (PAS)

\begin{tabular}{|c|c|c|c|}
\hline & $\begin{array}{l}\mathrm{HP} \\
(n=30)\end{array}$ & $\begin{array}{l}\text { PAS } \\
(n=30)\end{array}$ & $p$ Value \\
\hline \multicolumn{4}{|l|}{ Resection operation } \\
\hline Patients with complications & $18(60 \%)$ & $17(56 \%)$ & 1 \\
\hline Grade I & $2(7 \%)$ & $1(3 \%)$ & 0.83 \\
\hline Grade II & $2(7 \%)$ & $3(10 \%)$ & 0.83 \\
\hline Grade IIIa & $3(10 \%)$ & $4(13 \%)$ & 0.83 \\
\hline Grade IIIb & $3(10 \%)$ & $4(13 \%)$ & 0.83 \\
\hline Grade IVa & $2(7 \%)$ & $1(3 \%)$ & 0.83 \\
\hline Grade IVb & $1(3 \%)$ & $1(3 \%)$ & 0.83 \\
\hline Grade V & $5(17 \%)$ & $3(10 \%)$ & 0.83 \\
\hline Operative time (min) & $170 \pm 73$ & $270 \pm 79$ & $<0.001$ \\
\hline Blood loss (ml) & $400 \pm 501$ & $800 \pm 536$ & 0.04 \\
\hline Hospitalization (days) & 19 & 23 & 0.32 \\
\hline Stoma reversal operation ${ }^{a}$ & $15(60 \%)$ & $26(96 \%)$ & 0.001 \\
\hline Time to reversal (days) & 193 & 68 & 0.001 \\
\hline Patients with complications & $10(66 \%)$ & $6(23 \%)$ & 0.02 \\
\hline Grade I & $2(13 \%)$ & $1(4 \%)$ & 0.02 \\
\hline Grade II & $3(20 \%)$ & $2(8 \%)$ & 0.02 \\
\hline Grade IIIa & $1(7 \%)$ & $0(0 \%)$ & 0.02 \\
\hline Grade IIIb & $1(7 \%)$ & $1(4 \%)$ & 0.02 \\
\hline Grade Iva & $1(7 \%)$ & $0(0 \%)$ & 0.02 \\
\hline Grade IVb & $2(13 \%)$ & $1(4 \%)$ & 0.02 \\
\hline Grade V & $0(0 \%)$ & $0(0 \%)$ & 0.02 \\
\hline Operative time (min) & $240 \pm 105$ & $75 \pm 36$ & 0.002 \\
\hline Blood loss (ml) & $400 \pm 313$ & $40 \pm 81$ & 0.02 \\
\hline Hospitalization (days) & 15 & 6 & 0.002 \\
\hline
\end{tabular}

${ }^{a}$ Percentages refer to number of surviving patients after resection operation

Continuous variables are expressed in number of patients or mean \pm standard deviation

Complication grades: I, self-limiting; II, conservatively treated; III, treated under local (a) or general (b) anesthesia; IV, requiring ICU treatment due to single-organ (a) or multi-organ (b) failure; V, death

$355 \pm 109 \mathrm{~min} ; p=0.09)$, blood loss $(800 \pm 895 \mathrm{ml}$ versus $805 \pm 531 \mathrm{ml} ; p=1.00)$ and mean hospital stay (36 days versus 28 days; $p=0.17$ ) were not significantly different.

Is a protective Ileostomy required in case of primary anastomosis?

All 35 patients with PA (without protective stoma) operated during the same time period had purulent peritonitis (Hinchey 3); none had feculent peritonitis (Hinchey 4). For this reason, we matched all 17 PAS Hinchey 3 patients with 17 Hinchey 3 patients of the PA group. The PAS and PA patients were also well-matched one-to-one, for age, gender, ASA, BMI, and Hinchey stage. Moreover, the Mannheim Peritonitis Index (MPI) [17], APACHE II score
Table 3 Demographics of patients undergoing primary anastomosis without (PA) or with protective ileostomy (PAS)

\begin{tabular}{llll}
\hline & $\begin{array}{l}\text { PA } \\
(n=17)\end{array}$ & $\begin{array}{l}\text { PAS } \\
(n=17)\end{array}$ & $p$ Value \\
\hline Age (years) & $86 \pm 14$ & $67 \pm 18$ & 0.89 \\
Gender (male/ female) & $8 / 9$ & $7 / 10$ & 0.73 \\
BMI (kg/m²) & $25.4 \pm 4.0$ & $26.7 \pm 4.5$ & 0.06 \\
ASA I & 0 & 0 & \\
II & 4 & 6 & \\
III & 11 & 11 & \\
IV & 2 & 0 & \\
MPI & $18(10-29)$ & $21(12-36)$ & 0.63 \\
APACHE II & $11(5-19)$ & $13(1-23)$ & 0.68 \\
Immunosuppressed patients & $0 / 5$ & $0 / 5$ & 1 \\
$\quad$ transplant/ other) & & & \\
Etiology of perforation & $17 / 0 / 0$ & $14 / 3 / 0$ & 0.25 \\
$\quad$ diverticulitis/tumor/other) & & & \\
\hline
\end{tabular}

Data represent number of patients: mean \pm standard deviation for continuous data and median (range) of discrete data

[18], and number of immunosuppressed patients (5 versus 5) were comparable (Table 3 ).

Hospital mortality occurred in both groups: one patient in the PAS group and in two patients in the PA group. The complication rate of PA compared to PAS was similar (52\% versus $41 \% ; p=0.45$ ), as was the severity of complications $(p=0.51)$ (Table 4). Three patients in the PA group (18\%) and 4 patients in the PAS group (23\%) needed computed tomography (CT) guided drainage, surgical intervention, or ICU treatment (grade 3 and 4 complications). Two patients in each group developed an anastomotic leak (13\%). Including the procedure of stoma reversal, overall operative time and blood loss were not significantly different, but hospital stay was significantly shorter in PA, and no second operation was necessary. In contrast, there were two serious complications (two leakages, grade IIIb and IVb) after reversal of the diverting stoma.

\section{Discussion}

To our knowledge, this is the first case-matched comparison between the Hartmann operation and primary anastomosis for acute left-sided colon perforation in similar patient collectives, controlling for the most important risk factors of morbidity and mortality associated with these two surgical strategies. Not only the peritonitis stage according to Hinchey but also the severity of the septic disease assessed by MPI and APACHE II were similar in the two groups. First, our results demonstrate that primary anastomosis with protective ileostomy is superior to HP in 
Table 4 Primary anastomosis without (PA) or with protective ileostomy (PAS)

\begin{tabular}{|c|c|c|c|}
\hline & $\mathrm{PA}(n=17)$ & PAS $(n=17)$ & $p$ Value \\
\hline \multicolumn{4}{|l|}{ Resection operation } \\
\hline Patients with complications & $9(52 \%)$ & $7(41 \%)$ & 0.45 \\
\hline Grade I & 2 & 1 & 0.51 \\
\hline Grade II & 2 & 1 & 0.51 \\
\hline Grade IIIa & 1 & 2 & 0.51 \\
\hline Grade IIIb & 1 & 1 & 0.51 \\
\hline Grade IVa & 1 & 1 & 0.51 \\
\hline Grade IVb & 0 & 0 & 0.51 \\
\hline Grade V & 2 & 1 & 0.51 \\
\hline Operative time $(\min )$ & $170 \pm 87$ & $260.7 \pm 94.0$ & 0.16 \\
\hline Blood loss (ml) & $700 \pm 508$ & $926.7 \pm 658.2$ & 0.31 \\
\hline Hospitalization (days) & 17 & 23.8 & 0.029 \\
\hline \multicolumn{4}{|l|}{ Stoma reversal operation } \\
\hline Time to reversal (days) & & $55.9 \pm 52.7$ & \\
\hline $\begin{array}{l}\text { Patients with } \\
\text { complications }\end{array}$ & & 2 & \\
\hline \multicolumn{4}{|l|}{ Grade I } \\
\hline \multicolumn{4}{|l|}{ Grade II } \\
\hline Grade IIIa & Not applicable & & \\
\hline Grade IIIb & & 1 & \\
\hline \multicolumn{4}{|l|}{ Grade IVa } \\
\hline Grade IVb & & 1 & \\
\hline \multicolumn{4}{|l|}{ Grade V } \\
\hline Operative time (min) & Not applicable & & \\
\hline \multicolumn{4}{|l|}{ Blood loss (ml) } \\
\hline \multicolumn{4}{|l|}{ Hospitalization (days) } \\
\hline \multicolumn{4}{|l|}{ Both operations together } \\
\hline Operative time (min) & $169 \pm 87$ & $320 \pm 135$ & 0.003 \\
\hline Blood loss (ml) & $700 \pm 508$ & $950 \pm 618$ & 0.41 \\
\hline Hospitalization (days) & 17 & 28 & $<0.001$ \\
\hline
\end{tabular}

Complication grades: I, self limiting; II conservatively treated; III, treated under local (a) or general (b) anesthesia; IV, requiring ICU treament due to single-organ (a) or multi-organ (b) failure; V, death

Continuous variables are expressed in number of patients or mean \pm standard deviation

acute left-sided colon perforation with generalized peritonitis (purulent or feculent, Hinchey 3 or 4). While the total numbers and the severities of complications were similar after the resection operation, stoma reversal operations were more likely to be performed, and with significantly lower complications, in the primary anastomosis group. Second, we could show that primary anastomosis in cases of purulent peritonitis (Hinchey 3), i.e., excluding feculent peritonitis (Hinchey 4), does not require a protective ileostomy.

At present there are no convincing data in the literature on surgical strategies for acute left-sided colon perforation. The available studies are characterized by limited case numbers with discrepancies and weaknesses in the study design, as well as by selection bias, as demonstrated in a recent review of 98 studies [10]. Low-risk patients tend to be treated with PA, whereas high risk patients (aged over 65 years with co-morbidities or with severe peritonitis), are operated with HP [3-5]. This approach was also endorsed by the consensus conferences of the American Society of Colon and Rectal Surgeons [8] and the European Association of Endoscopic Surgeons [7]. The selection bias is most likely the main reason why results after HP are inferior to those after primary anastomosis [14, 20-22]. For $\mathrm{HP}$, mortality and morbidity rates have been described as high as $28 \%$ and up to $60 \%-70 \%$, respectively [11,23, 24], whereas reported complication rates for PAS are lower (mortality of approximately $10 \%$ and morbidity of $40 \%$ ) $[13,22,25,26]$.

Several prospective cohort studies have attempted to address the issue of selection bias, and treated all patients with perforated colonic disease with primary anastomosis $[12,27,28]$. Unfortunately, these studies were not revealing as the case numbers were low including mostly patients with localized peritonitis only (Hinchey 2).

The most important prognostic factors influencing morbidity and mortality are age, ASA score, obesity, the need for an emergency operation, and the severity of peritonitis (Hinchey stage or MPI index) [15, 25, 29-31]. In a recently published multivariate analysis of 156 patients, ASA score and preoperative organ failure were significantly associated with increased mortality [32]. The matching criteria used in our study covered these main prognostic factors well. Additionally the severity of the acute illness, assessed by MPI and APACHE II score, was similar in the two groups. Therefore HP and PAS patients could be compared. We found similar mortality rates associated with HP (17\%) and PAS (10\%), as well as the complication rates $(60 \%$ HP versus $56 \%$ PAS, death included) and complication severity, based on a therapyorientated complication score [19].

The main advantage of primary anastomosis with protective ileostomy compared to $\mathrm{HP}$ is the significant difference in the stoma reversal operation. In the present study, ileostomies were not only reversed more frequently (96\% versus 50\%), but earlier and with fewer and less severe complications. These figures are consistent with available data quoting reversal rates of $60 \%-70 \%$ for HP $[13,33]$ and $90 \%$ for PAS [10] and complications in up to $30 \%-55 \%$ of HP patients $[11,33,34]$ compared to $10 \%-$ $25 \%$ for ileostomy closure $[11,35,36]$.

With the demonstration of feasible and safe primary anastomosis in severe peritonitis, the next question is whether a diverting ileostomy is necessary. Recent studies of PA in the presence of acute colonic perforation describe complication rates ranging between $24 \%$ and $48 \%$ [12-14, 
$21,37]$, but most patients were at low risk with localized peritonitis, which constitutes a clear selection bias. The primary aim of a protective stoma in general peritonitis is to decrease the severity of potential anastomotic leakage, which occurs at a rate of $6.3 \%$ to $19.3 \%$ [10]. According to the review of Salem and Flum [10], patients undergoing primary anastomosis protected by a stoma present lower rates of leaks and wound infections compared to those without stoma. These rates are significantly higher than those observed in elective bowel surgery [38-40]. Because all patients with feculent peritonitis (Hinchey 4) in our collective were operated with protective ileostomy, we could only compare patients with purulent peritonitis (Hinchey 3 ) to assess the need for protective ileostomy. This comparison showed similar rates and severity of complications. The only differences between the two groups were longer total operative time $(+151 \mathrm{~min})$ and longer hospital stay (+11 days) for the PAS procedure (resection operation and stoma reversal taken together). The longer operating time for the first operation in the PAS group cannot be explained only by the time taken for the protective ileostomy. As these subgroups were well matched, the most likely explanation for the difference is technical difficulty, which may have influenced the surgeon toward protecting the anastomosis with a stoma. Moreover, stoma formation was a procedure commonly taught to residents. Anastomotic leakage occurred in two cases $(12 \%)$ in each group, suggesting that a diverting stoma may be unnecessary in the Hinchey 3 subgroup.

Although bowel preparation does not decrease the risk of leakage or infection in elective colon resection [41], the significance of intraoperative lavage [42] in minimizing the fecal load in cases of primary anastomosis in an emergency setting remains controversial. In experimental models of peritonitis, intraoperative colonic lavage had a positive effect on anastomotic healing [4345]. Clinical comparative trials evaluating the impact of intraopertive lavage in acute colorectal perforation do not exist; however, summarized results of noncomparative studies show better results for primary anastomosis with intraoperative lavage than without [10]. Moreover, in patients with protective ileostomies the evidence of intraoperative lavage is controversial. On the one hand, it does not seem logical to leave stool in the colon in cases of loop ileostomies. On the other hand, in most of the recently published trials on primary anastomosis with protective ileostomies showing the lowest rates of anastomotic leakages and reinterventions compared to studies including other procedures, intraoperative lavage was not performed [3, 9, 12, 46]. Protective stoma formation may influence the motility of the large bowel in terms of slowdown of passage with a potential protective effect on the healing of the anastomosis. Depending on the surgeon's preference, intraoperative colonic lavage in the present study was not routinely performed (PA: 9 cases of 17 [53\%]; PAS: 6 cases of 17 [35\%]). Interestingly, three of four anastomotic leakages (two in each group) were observed in patients with intraoperative colonic lavage, making the usefulness of this procedure questionable.

A possible limitation of the present study is that operations were performed at two different institutions, the first one recommending HP (80\%, academic center) and the second one PAS (73\%, large community hospital). Consequently, the operations were not performed by the same surgeons. The effect of "surgeon experience" on outcomes was not addressed by our analysis or by any of the previous published studies, but it may be relevant, as primary anastomosis is technically challenging in peritonitis. However, in both institutions, all procedures were performed under the supervision of a senior general surgeon, thus minimizing the effect of a learning curve or a lack of surgical skill. Another possible limitation concerns the influence of the underlying disease, including the severity of peritonitis, on the choice of the surgical procedure. Validated scores such as MPI and APACHE II were not used as matching parameters in the present study; however, they were equal in our patient collectives. The issue of severity of peritonitis has to be addressed precisely in any future study.

We conclude that primary anastomosis with protective ileostomy is superior to HP in perforated left-colon disease, even in the presence of severe generalized peritonitis (Hinchey 3 and 4). The complication rates after the resection operation were similar, but ileostomy closure was performed more frequently and was associated with significantly lower complication rates, less severe complications, and shorter hospitalization when compared to Hartmann reversal. Moreover, for Hinchey 3 peritonitis, protective ileostomy appears unnecessary, as there was no increased complication rate without stoma and no need for a second operation. As a next step a prospective randomized trial comparing HP with PAS is recommended to confirm that HP is replaced by PAS in cases of acute colon perforation.

Acknowledgments The authors thank Burkhart Seifert from the Institute of Biostatistics, University of Zurich, Switzerland, for his consultation.

\section{References}

1. Zeitoun G, Laurent A, Rouffet F, et al. (2000) Multicentre, randomized clinical trial of primary versus secondary sigmoid resection in generalized peritonitis complicating sigmoid diverticulitis. Br J Surg 87(10):1366-1374 
2. Krukowski ZH, Matheson NA (1984) Emergency surgery for diverticular disease complicated by generalized and faecal peritonitis: a review. Br J Surg 71(12):921-927

3. Zorcolo L, Covotta L, Carlomagno N, et al. (2003) Safety of primary anastomosis in emergency colo-rectal surgery. Colorectal Dis 5(3):262-269

4. Biondo S, Parés D, Marti Ragué J, et al. (2002) Emergency operations for nondiverticular perforation of the left colon. Am J Surg 183(3):256-260

5. Bielecki K, Kaminski P, Klukowski M (2002) Large bowel perforation: morbidity and mortality. Tech Coloproctol 6(3):177-182

6. Aldoori WH, Giovanucci EL, Rimm EB, et al. (1995) A prospective study of alcohol, smoking, caffeine, and the risk of symptomatic diverticular disease in men. Ann Epidemiol 5(3):221-228

7. Kohler L, Sauerland S, Neugebauer E (1999) Diagnosis and treatment of diverticular disease: results of a consensus development conference. The Scientific Committee of the European Association for Endoscopic Surgery. Surg Endosc 13(4):430-436

8. Wong WD, Wexner SD, Lowry A, et al. (2000) Practice parameters for the treatment of sigmoid diverticulitis-supporting documentation. The Standards Task Force. The American Society of Colon and Rectal Surgeons. Dis Colon Rectum 43(3):290-297

9. Schwesinger WH, Page CP, Gaskill HV III, et al. (2000) Operative management of diverticular emergencies: strategies and outcomes. Arch Surg 135(5):558-562; discussion 562-563

10. Salem L, Flum DR (2004) Primary anastomosis or Hartmann's procedure for patients with diverticular peritonitis? A systematic review. Dis Colon Rectum 47(11):1953-1964

11. Belmonte C, Klas JV, Perez JJ, et al. (1996) The Hartmann procedure. First choice or last resort in diverticular disease? Arch Surg 131(6):612-615; discussion 616-617

12. Gooszen AW, Gooszen HG, Veerman W, et al. (2001) Operative treatment of acute complications of diverticular disease: primary or secondary anastomosis after sigmoid resection. Eur J Surg 167(1):35-39

13. Regenet N, Pessaux P, Hennekinne S, et al. (2003) Primary anastomosis after intraoperative colonic lavage vs. Hartmann's procedure in generalized peritonitis complicating diverticular disease of the colon. Int J Colorectal Dis 18(6):503-507

14. Schilling MK, Maurer CA, Kollmar O, et al. (2001) Primary vs. secondary anastomosis after sigmoid colon resection for perforated diverticulitis (Hinchey stage III and IV): a prospective outcome and cost analysis. Dis Colon Rectum 44(5):699-703; discussion 703-705

15. Elliott TB, Yego S, Irvin TT (1997) Five-year audit of the acute complications of diverticular disease. Br J Surg 84(4):535-539

16. Hinchey EJ, Schaal PG, Richards GK (1978) Treatment of perforated diverticular disease of the colon. Adv Surg 12:85-109

17. Linder MM, Wacha H, Feldmann U, et al. (1987) [The Mannheim peritonitis index. An instrument for the intraoperative prognosis of peritonitis]. Chirurg 58(2):84-92

18. Knaus WA, Draper EA, Wagner DP, et al. (1985) APACHE II: a severity of disease classification system. Crit Care Med 13(10):818-829

19. Dindo D, Demartines N, Clavien PA (2004) Classification of surgical complications: a new proposal with evaluation in a cohort of 6336 patients and results of a survey. Ann Surg 240(2):205-213

20. Nespoli A, Ravizzini C, Trivella M, et al. (1993) The choice of surgical procedure for peritonitis due to colonic perforation. Arch Surg 128(7):814-818

21. Umbach TW Dorazio RA (1999) Primary resection and anastomosis for perforated left colon lesions. Am Surg 65(10):931-933
22. Wedell J, Banzhaf G, Choui R, et al. (1997) Surgical management of complicated colonic diverticulitis. Br J Surg 84(3):380 383

23. Berry AR, Turner WH, Mortensen NJMC, et al. (1989) Emergency surgery for complicated diverticular disease. A five-year experience. Dis Colon Rectum 32(10):849-854

24. Bokey EL, Chapuis PH, Fung C, et al. (1995) Postoperative morbidity and mortality following resection of the colon and rectum for cancer. Dis Colon Rectum 38(5):480-486; discussion 486-487

25. Zorcolo L, Covotta L, Carlomagno N, et al. (2003) Toward lowering morbidity, mortality, and stoma formation in emergency colorectal surgery: the role of specialization. Dis Colon Rectum 46(11):1461-1467; discussion 1467-1468

26. Biondo S, Perea MT, Ragué JM, et al. (2001) One-stage procedure in non-elective surgery for diverticular disease complications. Colorectal Dis 3(1):42-45

27. Hoemke M, Treckmann J, Schmitz R, et al. (1999) Complicated diverticulitis of the sigmoid: a prospective study concerning primary resection with secure primary anastomosis. Dig Surg 16(5):420-424

28. Maddern GJ, Nejjari Y, Dennison A, et al. (1995) Primary anastomosis with transverse colostomy as an alternative to Hartmann's procedure. Br J Surg 82(2):170-171

29. Hansen O, Graupe F, Stock W (1998) [Prognostic factors in perforating diverticulitis of the large intestine]. Chirurg 69(4):443-449

30. Maartense S, Vrancken Peeters MPFM, Spaander PJ, et al. (2003) Mortality after colon surgery: the value of a mortality registration system. Dig Surg 20(4):316-320

31. Makela JT, Kiviniemi H, Laitinen S (2005) Prognostic factors of perforated sigmoid diverticulitis in the elderly. Dig Surg 22(12):100-106

32. Biondo S, Ramos E, Deiros M, et al. (2000) Prognostic factors for mortality in left colonic peritonitis: a new scoring system. J Am Coll Surg 191(6):635-642

33. Mealy K, O’Broin E, Donohue J, et al. (1996) Reversible colostomy-what is the outcome? Dis Colon Rectum 39(11):1227-1231

34. Berne JD, Velmahos GC, Chan LS, et al. (1998) The high morbidity of colostomy closure after trauma: further support for the primary repair of colon injuries. Surgery 123(2):157-164

35. Gohring U, Lehner B, Schlag P (1988) [Ileostomy versus colostomy as temporary deviation stoma in relation to stoma closure]. Chirurg 59(12):842-844

36. Phang PT, Hain JM, Perez-Ramirez JJ, et al. (1999) Techniques and complications of ileostomy takedown. Am J Surg 177(6):463-466

37. Blair NP, Germann E (2002) Surgical management of acute sigmoid diverticulitis. Am J Surg 183(5):525-528

38. Illert B, Engemann R, Thiede A (2001) Success in treatment of complicated diverticular disease is stage related. Int J Colorectal Dis 16(5):276-279

39. Isbister WH, Prasad J (1997) Emergency large bowel surgery: a 15-year audit. Int J Colorectal Dis 12(5):285-290

40. Tang R, Hong $\mathrm{CH}$, Yung LW, et al. (2001) Risk factors for surgical site infection after elective resection of the colon and rectum: a single-center prospective study of 2,809 consecutive patients. Ann Surg 234(2):181-189

41. Guenaga KF, Lustosa SAS, Saad SS, et al. (2007) Ileostomy or colostomy for temporary decompression of colorectal anastomosis. Cochrane Database Syst Rev (1):CD004647

42. Dudley HA, Racliffe AG, McGeehan D (1980) Intraoperative irrigation of the colon to permit primary anastomosis. Br J Surg $67(2): 80-81$ 
43. Feres O, Monteiro dos Santos JC Jr, Andrade JI (2001) The role of mechanical bowel preparation for colonic resection and anastomosis: an experimental study. Int $\mathrm{J}$ Colorectal Dis 16(6):353-356

44. Ravo B, Metwall N, Yeh J (1991) Effect of fecal loading with/ without peritonitis on the healing of a colonic anastomosis: an experimental study. Eur Surg Res 23(2):100-107
45. Ravo B, Metwally N, Castera P, et al. (1988) The importance of intraluminal anastomotic fecal contact and peritonitis in colonic anastomotic leakages. An experimental study. Dis Colon Rectum 31(11):868-871

46. Landen S, Nafteux P (2002) Primary anastomosis and diverting colostomy in diffuse diverticular peritonitis. Acta Chir Belg 102(1):24-29 